\title{
Quantifying the impact of collider isolated photon data on global PDF fits
}

\author{
David d'Enterria* \\ CERN, PH Department, 1211 Geneva, Switzerland \\ E-mail: david.d'enterria@cern.ch
}

\section{Raphaëlle Ichou ${ }^{\dagger}$}

LPSC-Grenoble, 38026 Grenoble, France

E-mail: raphaelle.ichoudcern.ch

\begin{abstract}
We collect the world data of high- $p_{\mathrm{T}}$ isolated photon production in proton-(anti)proton collisions at collider energies $(\sqrt{s}=0.2-7 \mathrm{TeV})$ and show that they follow perturbative $x_{\mathrm{T}}$-scaling expectations. The 30 existing measurements are compared to next-to-leading-order perturbative QCD predictions using the JETPHOX code supplemented with the NNPDF2.1 parton densities. By applying the NNPDF reweighting technique one can quantify the additional constraints that these isolated photon datasets can impose on the parton distribution functions of the proton. This preliminary analysis suggest that present and future LHC isolated photon data could be useful in constraining the gluon density.
\end{abstract}

The 2011 Europhysics Conference on High Energy Physics-HEP 2011,

July 21-27, 2011

Grenoble, Rhône-Alpes France

\footnotetext{
* Speaker.

${ }^{\dagger}$ Currently at LPC (CNRS-IN2P3), Université Blaise Pascal, 63171 Clermont-Ferrand, France
} 


\section{Introduction}

Isolated prompt photon production in hadronic collisions at collider energies is directly sensitive to quark-gluon Compton scattering [1] and has been proposed since long [2, 3] to probe the gluon density in the proton which is otherwise only constrained indirectly by deep-inelastic data (and directly at high parton fractional momentum by inclusive jet production at Tevatron) [4]. Although there are about 30 isolated photon $p_{\mathrm{T}}$ spectra measured in $\mathrm{p}-\mathrm{p}$ and $\mathrm{p}-\overline{\mathrm{p}}$ collisions in the range $\sqrt{s}=0.2-7 \mathrm{TeV}$ at RHIC, Spp̄S, Tevatron and LHC (Table 1), these results (amounting to 353 data points) have not been included in global parton distribution functions (PDF) fits since more than 10 years [5]. The long-standing disagreement between next-to-leading order (NLO) perturbative QCD (pQCD) calculations [6] and the E706 inclusive photon spectra measured at $\sqrt{s} \approx 30 \mathrm{GeV}$ [7] motivated their removal from the PDF fit studies, although most of the E706 inclusive $\gamma$ production is dominated by uncertain parton-to-photon fragmentation contributions [8]. We show that (i) by imposing isolation criteria, and (ii) by increasing the $\sqrt{s}$ from fixed-target to collider energies, the fragmentation $\gamma$ contributions are significantly reduced [1] and the data-NLO agreement is very good for most of the measurements. A first study is presented, based on the JETPHOX code [6] and the NNPDF2.1 parton densities [9], to quantify the impact of adding all these isolated $\gamma$ data into global fits by using the NNPDF reweighting technique [10].

\section{Experimental data}
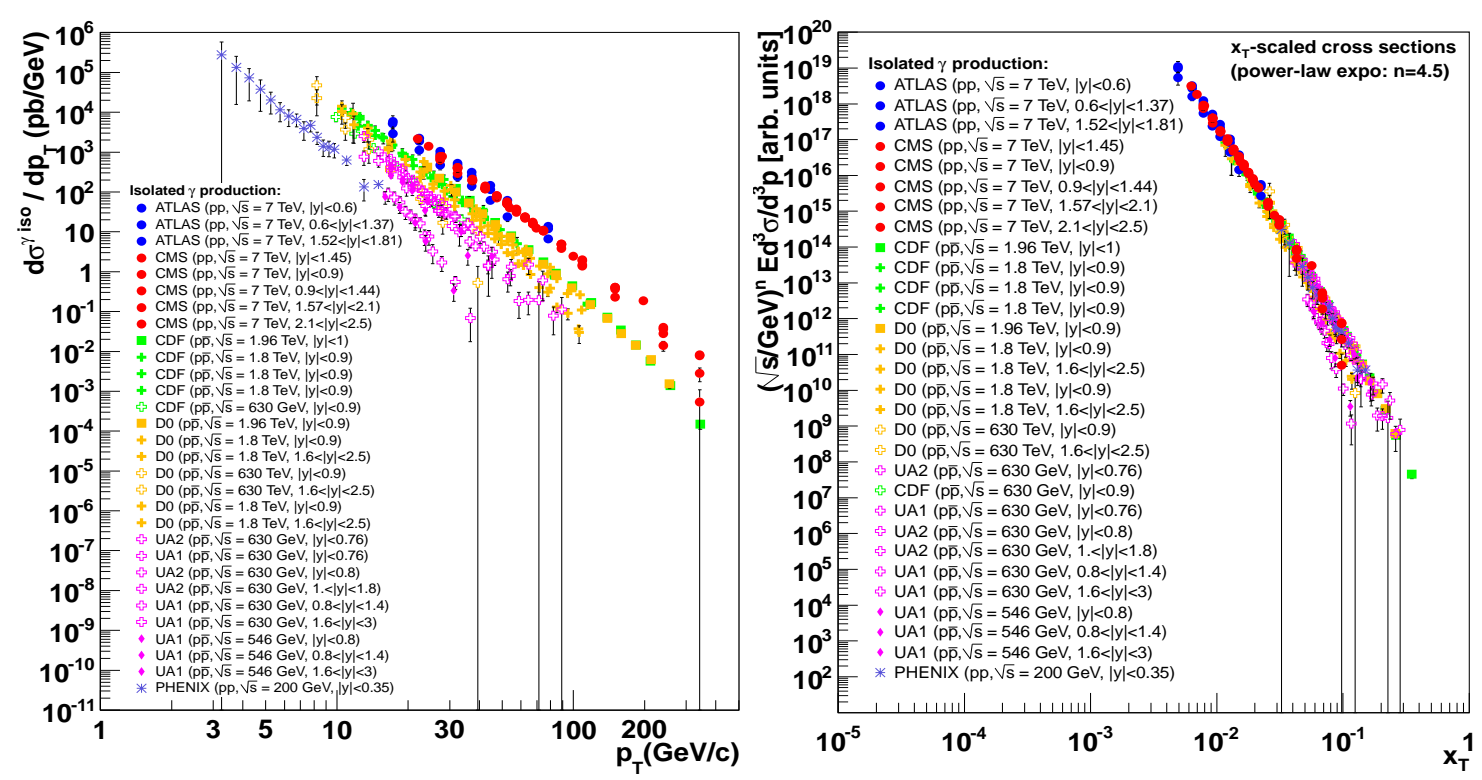

Figure 1: World systematics of isolated photon spectra measured as a function of $p_{\mathrm{T}}$ (left) and $x_{\mathrm{T}}$ (right, invariant cross sections scaled by $\sqrt{s}^{n}$ with $n=4.5$ ) in hadronic collisions at collider energies (Table 1).

The isolated photon data of Table 1 are plotted in Fig. 1 as a function of $p_{\mathrm{T}}$ (left panel) and as function of $x_{\mathrm{T}}=2 p_{\mathrm{T}} / \sqrt{s}$ with the cross sections scaled by $\sqrt{s}^{n}$ (right panel). All measured spectra follow clear power-law $p_{\mathrm{T}}$ dependencies from 3 to $400 \mathrm{GeV} / \mathrm{c}$ spanning 9 orders of magnitude 


\begin{tabular}{|c|c|c|c|c|c|c|c|}
\hline System & $\begin{array}{l}\text { Collab./Experiment } \\
\text { (collider) [Ref.] }\end{array}$ & $\begin{array}{l}\sqrt{s} \\
(\mathrm{TeV})\end{array}$ & $\begin{array}{c}y \mid \\
\text { range }\end{array}$ & $\begin{array}{l}p_{\mathrm{T}} \text { range } \\
(\mathrm{GeV} / \mathrm{c})\end{array}$ & $\begin{array}{c}x \\
\text { range }\end{array}$ & $\begin{array}{c}\text { Data } \\
\text { points }\end{array}$ & $\begin{array}{c}\text { Isolation } \\
\text { radius, had. energy }\end{array}$ \\
\hline p-p & ATLAS (LHC) [11] & 7. & $<0.6$ & $15 .-100$ & $5 \cdot 10^{-3}-0.05$ & 8 & $R=0.4, \mathrm{E}_{h}<5 \mathrm{GeV}$ \\
\hline p-p & ATLAS (LHC) [11] & 7. & $0.6-1.37$ & 15. -100 & $3 \cdot 10^{-3}-0.1$ & 8 & $R=0.4, \mathrm{E}_{h}<5 \mathrm{GeV}$ \\
\hline p-p & ATLAS (LHC) [11] & 7. & $1.52-1.81$ & 15. -100 . & $2 \cdot 10^{-3}-0.1$ & 8 & $R=0.4, \mathrm{E}_{h}<5 \mathrm{GeV}$ \\
\hline p-p & CMS (LHC) [12] & 7. & $<1.45$ & $21 .-300$ & $5 \cdot 10^{-3}-0.1$ & 11 & $R=0.4, \mathrm{E}_{h}<5 \mathrm{GeV}$ \\
\hline $\mathrm{p}-\mathrm{p}$ & CMS (LHC) [12] & 7. & $<0.9$ & $25 .-400$ & $5 \cdot 10^{-3}-0.2$ & 15 & $R=0.4, \mathrm{E}_{h}<5 \mathrm{GeV}$ \\
\hline $\mathrm{p}-\mathrm{p}$ & CMS (LHC) [12] & 7. & $0.9-1.44$ & $25 .-400$ & $2 \cdot 10^{-3}-0.3$ & 15 & $R=0.4, \mathrm{E}_{h}<5 \mathrm{GeV}$ \\
\hline p-p & CMS (LHC) [12] & 7. & $1.57-2.1$ & $25 .-400$ & $10^{-3}-0.4$ & 15 & $R=0.4, \mathrm{E}_{h}<5 \mathrm{GeV}$ \\
\hline $\mathrm{p}-\mathrm{p}$ & CMS (LHC) [12] & 7. & $2.1-2.5$ & $25 .-400$ & $10^{-3}-0.5$ & 15 & $R=0.4, \mathrm{E}_{h}<5 \mathrm{GeV}$ \\
\hline $\mathrm{p}-\overline{\mathrm{p}}$ & CDF (Tevatron) [13] & 1.96 & $<1.0$ & $30 .-400$ & $0.01-0.4$ & 16 & $R=0.4, \varepsilon_{h}=0.1$ \\
\hline $\mathrm{p}-\overline{\mathrm{p}}$ & D0 (Tevatron) [14] & 1.96 & $<0.9$ & 23. -300 & $0.01-0.3$ & 17 & $R=0.4, \mathrm{E}_{h}<2 \mathrm{GeV}$ \\
\hline $\mathrm{p}-\overline{\mathrm{p}}$ & CDF (Tevatron) [15] & 1.8 & $<0.9$ & 8. - 132 & $5 \cdot 10^{-3}-0.2$ & 16 & $R=0.7, \mathrm{E}_{h}<2 \mathrm{GeV}$ \\
\hline $\mathrm{p}-\overline{\mathrm{p}}$ & CDF (Tevatron) [16] & 1.8 & $<0.9$ & 11. -132 . & $5 \cdot 10^{-3}-0.2$ & 17 & $R=0.4, \mathrm{E}_{h}<4 \mathrm{GeV}$ \\
\hline$p-\bar{p}$ & CDF (Tevatron) [17] & 1.8 & $<0.9$ & 10. -65 & $5 \cdot 10^{-3}-0.1$ & 17 & $R=0.4, \mathrm{E}_{h}<1 \mathrm{GeV}$ \\
\hline $\mathrm{p}-\overline{\mathrm{p}}$ & D0 (Tevatron) [18] & 1.8 & $<0.9$ & 9. - 126 & $5 \cdot 10^{-3}-0.2$ & 23 & $R=0.4, \mathrm{E}_{h}<2 \mathrm{GeV}$ \\
\hline $\mathrm{p}-\overline{\mathrm{p}}$ & D0 (Tevatron) [18] & 1.8 & $1.6-2.5$ & 9. - 126 & $10^{-3}-0.4$ & 23 & $R=0.4 \mathrm{E}_{h}<2 \mathrm{GeV}$ \\
\hline $\mathrm{p}-\overline{\mathrm{p}}$ & D0 (Tevatron) [19] & 1.8 & $<0.9$ & 10. -140 & $5 \cdot 10^{-3}-0.2$ & 9 & $R=0.4, \mathrm{E}_{h}<2 \mathrm{GeV}$ \\
\hline $\mathrm{p}-\overline{\mathrm{p}}$ & D0 (Tevatron) [19] & 1.8 & $1.6-2.5$ & 10. -140 & $10^{-3}-0.4$ & 9 & $R=0.4, \mathrm{E}_{h}<2 \mathrm{GeV}$ \\
\hline $\mathrm{p}-\overline{\mathrm{p}}$ & CDF (Tevatron) [16] & 0.63 & $<0.9$ & 8. -38 . & $0.01-0.2$ & 7 & $R=0.4, \mathrm{E}_{h}<4 \mathrm{GeV}$ \\
\hline $\mathrm{p}-\overline{\mathrm{p}}$ & D0 (Tevatron) [20] & 0.63 & $<0.9$ & 7. -50 . & $0.01-0.3$ & 7 & $R=0.4, \mathrm{E}_{h}<2 \mathrm{GeV}$ \\
\hline $\mathrm{p}-\overline{\mathrm{p}}$ & D0 (Tevatron) [20] & 0.63 & $1.6-2.5$ & 7. -50 . & $10^{-3}-0.4$ & 7 & $R=0.4, \mathrm{E}_{h}<2 \mathrm{GeV}$ \\
\hline $\mathrm{p}-\overline{\mathrm{p}}$ & UA1 (Sp̄̄S) [21] & 0.63 & $<0.8$ & $16 .-100$ & $0.03-0.3$ & 16 & $R=0.7, \mathrm{E}_{h}<2 \mathrm{GeV}$ \\
\hline $\mathrm{p}-\overline{\mathrm{p}}$ & UA1 (Sp̄̄s) [21] & 0.63 & $0.8-1.4$ & 16. -70 & $0.01-0.4$ & 10 & $R=0.7, \mathrm{E}_{h}<2 \mathrm{GeV}$ \\
\hline $\mathrm{p}-\overline{\mathrm{p}}$ & UA1 (Spp̄S) [21] & 0.63 & $1.6-3$. & 16. -70 & $0.01-0.5$ & 13 & $R=0.7, \mathrm{E}_{h}<2 \mathrm{GeV}$ \\
\hline $\mathrm{p}-\overline{\mathrm{p}}$ & UA2 (Spp̄S) [22] & 0.63 & $<0.76$ & 14. -92 & $0.03-0.3$ & 13 & $R=0.265, \varepsilon_{h}=0.25$ \\
\hline $\mathrm{p}-\overline{\mathrm{p}}$ & UA2 (Sp̄̄s) [23] & 0.63 & $<0.76$ & 12. -83 & $0.03-0.3$ & 14 & $R=0.25, \mathrm{E}_{h}<0.1 \mathrm{GeV}$ \\
\hline $\mathrm{p}-\overline{\mathrm{p}}$ & UA2 (Sp̄̄s) [23] & 0.63 & 1. -1.8 & 12. -51 & $0.01-0.4$ & 8 & $R=0.53, \mathrm{E}_{h}<2 \mathrm{GeV}$ \\
\hline $\mathrm{p}-\overline{\mathrm{p}}$ & UA1 (Sp̄̄S) [21] & 0.546 & $<0.8$ & 16. -51 & $0.03-0.2$ & 6 & $R=0.7, \mathrm{E}_{h}<2 \mathrm{GeV}$ \\
\hline $\mathrm{p}-\overline{\mathrm{p}}$ & UA1 (Spp̄S) [21] & 0.546 & $0.8-1.4$ & 16. -46 & $0.02-0.4$ & 5 & $R=0.7, \mathrm{E}_{h}<2 \mathrm{GeV}$ \\
\hline $\mathrm{p}-\overline{\mathrm{p}}$ & UA1 (Spp̄S) [21] & 0.546 & $1.6-3$ & 16. -38 & $0.01-0.5$ & 5 & $R=0.7, \mathrm{E}_{h}<2 \mathrm{GeV}$ \\
\hline p-p & PHENIX (RHIC) [24] & 0.2 & $<0.35$ & 3. -16 . & $0.03-0.2$ & 17 & $R=0.5, \varepsilon_{h}=0.1$ \\
\hline
\end{tabular}

Table 1: World systematics of isolated photon data in p-p and $p-\bar{p}$ collisions. For each system, we quote (i) the experiment and bibliographical reference, (ii) center-of-mass energy, measured (iii) rapidity and (iv) $p_{\mathrm{T}}$ ranges, (v) parton fractional momenta probed, (vi) number of data points, and the (v) isolation criteria used.

in the cross section. The $x_{\mathrm{T}}$ spectra coalesce over a single curve ${ }^{1}$ when the $\sqrt{s}$-dependent cross sections are scaled with a power-law exponent $n \approx 4.5$ very close to the expected $n=4$ conformal limit for partonic $2 \rightarrow 2$ scattering [25]. These observations are tell-tale of a perturbative origin for the production of isolated photons and justify the use of pQCD calculations to study their yields.

\section{Theoretical setup and results}

We run the JETPHOX code at NLO accuracy with the NNPDF2.1 parton densities interfaced via the LHAPDF 5.8.5 package [26] for each one of the measurements listed in Table 1 taking into account each individual kinematics and isolation cuts. We set the renormalization, factorization and fragmentation scales to a default $\mu=p_{\mathrm{T}}$, and use the BFG-II ("large gluon") parton-tophoton fragmentation functions [8]. By running over a hundred PDF replicas for each system and computing the $\chi^{2}$ (and associated weight) of each replica with respect to the data, one can obtain a set of reweighted replicas and quantitatively estimate the additional constraints that the world

\footnotetext{
${ }^{1}$ A few deviations are visible for measures away from midrapidity for which $x_{\mathrm{T}}=2 p_{\mathrm{T}} / \sqrt{s}$ does not exactly hold.
} 
isolated $\gamma$ data can impose on the parton distributions of the proton. Preliminary results of the procedure (about $2 / 3$ of the measurements have all replicas with $\chi^{2} / \mathrm{N}_{\text {points }}<2$ while the rest have $\chi^{2} / \mathrm{N}_{\text {points }} \approx 2-4$ ) suggest that present and future LHC isolated photon data could be useful in imposing extra independent constraints on the gluon density in the fractional momentum range $x \approx 0.005-0.1$.

Acknowledgments: We are grateful to F. Arleo and J.Ph. Guillet for useful feedback on JETPHOX, as well as to J. Rojo and G. Watt for valuable discussions on PDF fits.

\section{References}

[1] R. Ichou, D. d'Enterria, Phys. Rev. D 82 (2010) 014015

[2] P. Aurenche, R. Baier, M. Fontannaz, J. F. Owens and M. Werlen, Phys. Rev. D 39 (1989) 3275

[3] W. Vogelsang and A. Vogt, Nucl. Phys. B 453 (1995) 334

[4] A. De Roeck, R. S. Thorne, Prog. Part. Nucl. Phys. 66 (2011) 727

[5] A. D. Martin, R. G. Roberts, W. J. Stirling and R. S. Thorne, Eur. Phys. J. C 14 (2000) 133

[6] P. Aurenche, M. Fontannaz, J. P. Guillet, E. Pilon and M. Werlen, Phys. Rev. D 73 (2006) 094007; http://lappweb.in2p3.fr/lapth/PHOX_FAMILY/jetphox.html

[7] L. Apanasevich et al. [E706 Collab.], Phys. Rev. Lett. 81 (98) 2642; Phys. Rev. D 70 (2004) 092009

[8] L. Bourhis, M. Fontannaz and J. P. Guillet, Eur. Phys. J. C 2 (1998) 529

[9] R. D. Ball et al. [NNPDF Collab.], Nucl. Phys. B 849 (2011) 296

[10] R. D. Ball et al. [NNPDF Collab.], Nucl. Phys. B 849 (2011) 112 [Erratum-ibid. B 854 (2012) 926]

[11] G. Aad et al. [ATLAS Collab.], Phys. Rev. D 83 (2011) 052005

[12] V. Khachatryan et al. [CMS Collab.], Phys. Rev. Lett. 106 (11) 082001; Phys. Rev. D84 (11) 052011

[13] T. Aaltonen et al. [CDF Collab.], Phys. Rev. D 80 (2009) 111106

[14] V. M. Abazov et al. [D0 Collab.], Phys. Lett. B 639 (2006) 151 [Erratum-ibid. B 658 (2008) 285]

[15] F. Abe et al. [CDF Collab.], Phys. Rev. Lett. 73 (1994) 2662 [Erratum-ibid. 74 (1995) 1891].

[16] D. E. Acosta et al. [CDF Collab.], Phys. Rev. D 65 (2002) 112003

[17] D. E. Acosta et al. [CDF Collab.], Phys. Rev. D 70 (2004) 074008

[18] S. Abachi et al. [D0 Collab.], Phys. Rev. Lett. 77 (1996) 5011

[19] B. Abbott et al. [D0 Collab.], Phys. Rev. Lett. 84 (2000) 2786

[20] V. M. Abazov et al. [D0 Collab.], Phys. Rev. Lett. 87 (2001) 251805

[21] C. Albajar et al. [UA1 Collab.], Phys. Lett. B 209 (1988) 385

[22] J. Alitti et al. [UA2 Collab.], Phys. Lett. B 288 (1992) 386

[23] R. Ansari et al. [UA2 Collab.], Z. Phys. C 41 (1988) 395

[24] S. S. Adler et al. [PHENIX Collab.], Phys. Rev. Lett. 98 (2007) 012002

[25] F. Arleo, S. J. Brodsky, D. S. Hwang, A. M. Sickles, Phys. Rev. Lett. 105 (2010) 062002

[26] M. R. Whalley, D. Bourilkov and R. Group, arXiv:hep-ph/0508110; 